Check for updates

Received 26th March 2018

DOI: $10.1039 / c 8 r a 02595 a$

rsc.li/rsc-advances
Cite this: RSC Adv., 2018, 8, 18745

Accepted 9th May 2018

\section{Zein regulating apatite mineralization, degradability, in vitro cells responses and in vivo osteogenesis of 3D-printed scaffold of $n-M S / Z N /$ PCL ternary composite}

\author{
Jiangying Ru, ${ }^{a}$ Qiang Wei, ${ }^{\mathrm{b}}$ Lianqing Yang, ${ }^{\mathrm{b}}$ Jing Qin, ${ }^{\mathrm{c}}$ Liangchen Tang, (D) ${ }^{\mathrm{c}} \mathrm{Jie} \mathrm{Wei}^{\mathrm{c}}$ \\ Lieping Guo*d and Yunfei Niu (D)*b
}

\begin{abstract}
Bioactive and degradable scaffolds of nano magnesium silicate (n-MS)/zein (ZN)/poly(caprolactone) (PCL) ternary composites were prepared by 3D-printing method. The results showed that the 3D-printed scaffolds possessed controllable pore structure, and pore morphology, pore size, porosity and pore interconnectivity of the scaffolds can be efficiently adjusted. In addition, the apatite-mineralization ability of the scaffolds in simulated body fluids was obviously improved with the increase of $Z N$ content, in which the scaffold with 20 w\% ZN (C20) possessed excellent apatite-mineralization ability. Moreover, the degradability of the scaffolds was significantly enhanced with the increase of $Z N$ content in the scaffolds. The degradation of ZN produced acidic products that could neutralize the alkaline products from the degradation of $\mathrm{n}-\mathrm{MS}$, which avoid the increase of $\mathrm{pH}$ value in degradable solution. Furthermore, the MC3T3-E1 cells responses (e.g. proliferation and differentiation, etc.) to the scaffolds were significantly promoted with the increase of ZN content. The in vivo osteogenesis of the scaffolds implanted the femur defects of rabbits was investigated by micro-CT and histological analysis. The results demonstrated that the new bone formation was significantly enhanced with the increase of ZN content, in which the $\mathrm{C} 20$ scaffold induced the highest new bone tissues, indicating excellent osteogenesis. The results suggested that the $\mathrm{ZN}$ in the ternary composite scaffolds played key roles in assisting bone regeneration in vivo.
\end{abstract}

\section{Introduction}

Over the past few decades, bioactive scaffolds of polymer based biocomposites containing bioactive materials (e.g. hydroxyapatite, tricalcium phosphate, bioglass, and calcium silicate, etc.) and degradable polymers (e.g. poly(lactic acid), poly(glycolic acid), poly(lactic-co-glycolic acid) and poly(caprolactone), etc.) with great potential for tissue engineering scaffolds have been widely reported..$^{1-3}$ The polymer based biocomposites scaffolds have been fabricated by means of different conventional technologies, including rapid prototyping, solvent casting and particulate-leaching, gas foaming, foam replica method, electrospinning, freeze-drying and phase separation, etc. ${ }^{4-6}$ The advantages of these conventional methods are inexpensive and

${ }^{a}$ Department of Orthopaedics, The Affiliated Hospital of Yangzhou University, Yangzhou University, Yangzhou 225009, China

${ }^{b}$ Department of Orthopaedics, Changhai Hospital, Second Military Medical University, Shanghai 200433, China. E-mail: niuyunfei1219@163.com

'Key Laboratory for Ultrafine Materials of Ministry of Education, East China University of Science and Technology, Shanghai 200237, China

${ }^{d}$ Department of Oncology, Shanghai Eastern Hepatobiliary Surgery Hospital, Shanghai, 200438, China. E-mail: glplaying2000@163.com simple, which are flexible to optimize or control physicalchemical and biological performances of the scaffolds. However, the main disadvantages of these technologies are that they cannot efficiently modulate the porous structures of the scaffolds, such as pore size, porosity, pore morphology, and pore interconnectivity, etc. ${ }^{7}$

Synthetic polymer of biodegradable poly(caprolactone) (PCL) with good biocompatibility and mechanical strengths has been applied in biomedical fields for many years. ${ }^{8}$ However, the significant disadvantages of PCL are that PCL is bioinert materials (no inducing new bone regeneration) and its degradability is very slow both in vitro and in vivo. ${ }^{9}$ Natural polymer of plant proteins (e.g. zein, soy proteins and wheat protein, etc.) have attracted more and more interests for biomedical materials. ${ }^{10}$ Due to the excellent biocompatibility and biodegradability, biomaterials developed from plant proteins are preferred for biomedical applications over synthetic polymers. ${ }^{11}$ One of plant proteins of zein (ZN) with good biocompatibility have been investigated for various applications, such as deoxyribonucleic acid (DNA) transfection, vaccine delivery, oral delivery of proteins and peptides, and tissue engineering scaffolds. ${ }^{12,13}$ The advantage of $\mathrm{ZN}$ is its fast 
biodegradability while the disadvantage of $\mathrm{ZN}$ is its low mechanical strength. ${ }^{12-14}$

Previous study reported that nano magnesium silicate (nMS) bioglass exhibited superior in vitro bioactivity (apatite formation), cytocompatibility and in vivo osteogenesis properties as compared with traditional magnesium silicate bioglass. ${ }^{15,16}$ However, the main disadvantages of n-MS are its inherent brittleness and low mechanical strength. ${ }^{15,16}$ Combination of the advantages of both biodegradable polymers and bioactive materials would cause the composites scaffolds with improved bioperformances (e.g. bioactivity, degradability and mechanical strength, etc. $){ }^{\mathbf{1 7}}$

Recently, the scaffolds fabricated by 3D-printing have been utilized for application in bone tissue engineering. ${ }^{18}$ The significant advantages of this method are that the porous structures (pore morphology, pore size, porosity and interconnectivity, etc.) of the scaffolds can be concisely controlled by layer-by-layer plotting under different conditions. ${ }^{18,19}$ Therefore, in this study, nano magnesium silicate (n-MS) particles were fabricated, and bioactive and degradable scaffolds of n-MS/ZN/ PCL ternary composites with controllable pore structure were developed by 3D printing method. The objective of this study to investigate the effects of $\mathrm{ZN}$ content in the scaffolds on in vitro apatite mineralization, degradability, cell responses and in vivo osteogenesis properties of the ternary composites scaffolds.

\section{Materials and methods}

\section{Preparation and characterization of nano magnesium silicate}

The nano magnesium silicate (n-MS) were synthesized by a solgel method using cetyltrimethylammonium bromide (CTAB) as the template, in which $6.6 \mathrm{~g}$ CTAB was firstly dissolved in $600 \mathrm{~mL}$ distilled water, then $12 \mathrm{~mL}$ ammonia aqueous solution $(28 \mathrm{w} \%)$ was added into the CTAB solution. After stirring for $1 \mathrm{~h}$, $30 \mathrm{~mL}$ tetraethyl orthosilicate (TEOS, 98\%) and $17.2 \mathrm{~g}$ $\mathrm{Mg}\left(\mathrm{NO}_{3}\right)_{2} \cdot 6 \mathrm{H}_{2} \mathrm{O}$ were added into the solution and stirred for $6 \mathrm{~h}$. The products were collected by vacuum filtration, and washed by distilled water and ethanol for 3 times, respectively, then dried at $60{ }^{\circ} \mathrm{C}$ for $24 \mathrm{~h}$. The resulting powders were then calcined at $550{ }^{\circ} \mathrm{C}$ for $2 \mathrm{~h}$ to remove the CTAB, and the final nMS powders were obtained. ${ }^{15,16}$ All raw materials used in this process were purchased from Shanghai Lingfeng Chemical Reagent Co., Ltd., China. The n-MS powders were characterized by transmission electron microscopy (TEM, 2100F, JEOL, Japan), EDS (TEM, 2100F, JEOL, Japan) and X-ray diffraction (XRD, D/Max 2550V, Rigaku, Japan).

\section{Preparation and characterization of ternary composites scaffolds}

The 3D Bioplotter ${ }^{\mathrm{TM}}$ printing device (EnvisionTEC $\mathrm{GmbH}$, Germany) was used to prepare 3D-printed scaffolds. Prior to printing the scaffolds, the injectable n-MS/ZN/PCL paste was prepared as following: $2 \mathrm{~g}$ mixture containing $\mathrm{n}-\mathrm{MS}, \mathrm{ZN}$ and PCL was dispersed into $20 \mathrm{~mL}$ chloroform according to the component proportion listed in Table 1 . The dispersion was then quickly stirred at room temperature until it formed a paste for
Table 1 Composition of scaffolds prepared by 3D printing method

\begin{tabular}{llll}
\hline Samples & C0 & C10 & C20 \\
\hline ZN & 0 & $10 \mathrm{w} \%$ & $20 \mathrm{w} \%$ \\
PCL & $70 \mathrm{w} \%$ & $60 \mathrm{w} \%$ & $50 \mathrm{w} \%$ \\
n-MS & $30 \mathrm{w} \%$ & $30 \mathrm{w} \%$ & $30 \mathrm{w} \%$ \\
\hline
\end{tabular}

injection. Finally, the prepared paste was introduced into a polyethylene injection cartridge, which was fixed onto the device. Simultaneously, square block models $(6 \times 6 \times 6 \mathrm{~mm})$ were loaded on the Bioplotter ${ }^{\mathrm{TM}} \mathrm{CAD} / \mathrm{CAM}$ software, and the scaffolds were plotted layer-by-layer through the extrusion of the paste. The architecture was changed by plotting fibers with 0 and 90 angle steps between two successive layers; the dosing pressure to the syringe pump was 1.5-3.0 bar, the speed of the dispensing unit was $1.5-5 \mathrm{~mm} \mathrm{~s}^{-1}$, and the nozzle size was 0.25 $\mathrm{mm}$. Finally, the obtained n-MS/ZN/PCL scaffolds (C0, C10, C20) were dried in an oven at $37{ }^{\circ} \mathrm{C}$ for 2 days (evaporate chloroform).

The as-prepared scaffolds were characterized by scanning electron microscope (SEM, S-4800, Hitachi, Japan) and XRD. The porosity of the 3D-printed scaffolds was evaluated by using Archimedes method: porosity $=\left(W_{\mathrm{b}}-W_{\mathrm{a}}\right) /\left(W_{\mathrm{b}}-W_{\mathrm{c}}\right) \times 100 \%$, where $W_{\mathrm{a}}$ was the dry weight of the samples, $W_{\mathrm{b}}$ was the wet weight of the scaffolds and $W_{\mathrm{c}}$ was the weight of the scaffolds suspended in water. The compressive strength of the 3D-printed scaffolds was evaluated by using a mechanical testing machine (HY-0230, Shanghai, China).

\section{Apatite mineralization of scaffolds in SBF}

The assessment of in vitro bioactivity of the scaffolds was carried out in simulated body fluids (SBF) solution. Each scaffold was soaked in $10 \mathrm{~mL}$ of SBF solution in a polyethylene bottle at $37{ }^{\circ} \mathrm{C}$ for different time. At each time interval, the scaffolds were collected, washed gently with distilled water, and dried at $60{ }^{\circ} \mathrm{C}$. SEM and EDS were used to examine the mineralized apatite on scaffolds surfaces. The ions concentrations ( $\mathrm{Mg}, \mathrm{Si}, \mathrm{Ca}$ and $\mathrm{P}$ ) in solution after the scaffolds soaking for different time were determined by ICP-AES (Perkin-Elmer Optima 7000DV).

\section{Degradation of scaffolds in PBS}

To evaluation the degradable properties of the scaffolds, the weight change of the samples before and after soaking into phosphate buffer saline (PBS) for up to 84 days was monitored by the electronic analytical balance (AL204, Mettler Toledo, Switzerland), and the weight change ratio was calculated according to the following equation: ${ }^{18}$ weight change $(\%)=\left(W_{n}\right.$ - $\left.W_{0}\right) / W_{0}$, where $W_{0}$ represents the samples weight before soaking and $W_{n}$ represents samples weight after soaking for different times. In addition, the change of $\mathrm{pH}$ value in solution for the scaffolds during the immersion period was also measured by a pH meter (FE20K, Mettler Toledo, Switzerland) at designated points of time. 


\section{In vitro cytocompatibility of scaffolds}

Cells culture. MC3T3-E1 cells, an osteoblast precursor cell line derived from mouse (Cell Culture Center, Chinese Academy of Medical Science, China), was used to evaluate influence of the scaffolds on cell proliferation and differentiation. The cells were cultured in Dulbecco's Modified Eagle Medium (DMEM, Gibco, USA) supplemented with $10 \%$ fetal bovine serum (FBS, Gibco, ThermoFisher Scientific, USA), $100 \mathrm{U} \mathrm{mL}^{-1}$ penicillin and 100 $\mu \mathrm{g} \mathrm{mL}{ }^{-1}$ streptomycin sulphate at $37{ }^{\circ} \mathrm{C}$ in a humidified atmosphere of $5 \% \mathrm{CO}_{2}$. Then the cells were detached with $0.25 \%$ trypsin, $0.03 \%$ ethylene diamine tetraacetic acid (EDTA) and the cell density was counted. After counting, the cell suspension was diluted to the desired density for following cell experiments. After sterilized by ethylene oxide, the scaffolds were placed into 24-well plate and co-cultured with the cells with density of $1 \times 10^{5}$ cells per well for different time.

Cells morphology and proliferation. After culturing for $6 \mathrm{~h}$, the scaffolds with the cells were fixed in $4 \%$ paraformaldehyde for $30 \mathrm{~min}$, and rinsed with phosphate-buffered saline (PBS) for 3 times. Moreover, the samples were treated with $0.1 \%$ Triton X100 to permeabilize the cells for $20 \mathrm{~min}$ and then blocked with $1 \%$ BSA for $20 \mathrm{~min}$. Finally, the actin cytoskeletons were labeled by incubating with Phalloidin-FITC (Sigma, USA) for $30 \mathrm{~min}$, and the cell nuclei were contrast-labeled in blue by $4^{\prime} 6$ diamidino-2-phenylindole dihydrochloride (DAPI, Sigma, USA) for $5 \mathrm{~min}$. The actin cytoskeletons of cells were visualized with a confocal laser scanning microscope (CLSM, Leica, Germany).

For the cell proliferation assay, the cells were cultured on the samples in 24-well plates for 1, 3 and 5 days. ${ }^{19}$ Three pieces of co-cultured discs for each group were washed twice with PBS, $400 \mu \mathrm{L}$ DMEM with supplement $40 \mu \mathrm{L} 5 \mathrm{mg} \mathrm{mL}{ }^{-1}$ MTT (Amresco, USA) solution was added and incubated at $37^{\circ} \mathrm{C}$ for $4 \mathrm{~h}$ to form MTT formazan. Finally, the medium was replaced with $400 \mu \mathrm{L}$ dimethyl sulfoxide (DMSO, Sigma, USA) in order to dissolve the formazan, while the absorbance was measured at $490 \mathrm{~nm}$ using an ELX Ultra Microplate Reader (Bio-tek, USA).

Alkaline phosphatase (ALP) activity. The ALP activity was determined as previously described at day 7, 10 and 14 after cell culturing with scaffolds. ${ }^{19}$ Briefly, the cells were collected and resuspended in lysis buffer with $0.2 \%$ NP-40. Each scaffold was mixed with $p$-nitrophenyl phosphate ( $p$ NPP, $1 \mathrm{mg} \mathrm{mL}^{-1}$, Sigma, USA) in $1 \mathrm{M}$ diethanolamine buffer and incubated at $37^{\circ} \mathrm{C}$ for $15 \mathrm{~min}$. Then, the reaction was stopped by the addition of $3 \mathrm{~N}$ $\mathrm{NaOH}$ to the reaction mixture. Finally, ALP activity was quantified by ELX Ultra Microplate Reader with absorbance at $405 \mathrm{~nm}$, while total protein content was determined with the Bradford method in aliquots of the same samples with the BioRad protein assay kit (Bio-Rad, USA), read at $630 \mathrm{~nm}$ and calculated according to a series of BSA (Sigma, USA) standards. ALP activity was expressed as absorbance at $405 \mathrm{~nm}$ (OD value) per milligram of total cellular proteins.

\section{In vivo osteogenesis of scaffolds}

Animal surgery. The female New Zealand white rabbit (3 months in age and around 2.8 in weight) were used according to guidelines (NIH guidelines for the care and use of laboratory animals (NIH Publication no. 85-23 Rev. 1985)), which were approved by the Animal Experiment and Care Committee of Ninth People's Hospital affiliated to Shanghai Jiao Tong University School of Medicine. Briefly, the 9 rabbits were randomly divided into 3 groups, and anesthetized with intramuscular injection of sodium pentobarbital $\left(20 \mathrm{mg} \mathrm{kg} \mathrm{kg}^{-1}\right.$, Sigma, USA). Then an incision with length of $1 \mathrm{~cm}$ was created to expose the lateral site of right femoral condyle of each rabbit, and a medical high-speed bur was used to drill holes $(6 \mathrm{~mm}$ in diameter, $6 \mathrm{~mm}$ in depth) and C0, C10 and C20 scaffolds were inserted into holes immediately. Subsequently, the incisions were closed with sutures. At week 4 and 12 after surgery, the rabbits in the corresponding group were sacrificed by an overdose of pentobarbital sodium, and the bone samples containing scaffolds were harvested and fixed by $2.5 \%$ glutaraldehyde solution.

Micro-computed tomography (m-CT) analysis. The bone samples were examined by a micro-CT system (mCT-80, Scanco Medical AG, Switzerland) with spot size of $20 \mathrm{~mm}$ and maximum voltage of $36 \mathrm{kV}$. After micro-CT scan, the $2 \mathrm{D}$ images of all bone samples were integrated by Adobe Photoshop CS6 Software (Adobe System, USA).

Histological analysis. After m-CT evaluation, the bone samples were decalcified using $15 \%$ ethylenediaminetetraacetic acid (EDTA, Fluka BioChemika, Sigma-Aldrich), bisected in the longitudinal axis using the lateral border of the intercondylar notch as a guide, and processed using an automated tissue processor (ASP300, Leica, Germany) before being embedded in paraffin wax blocks. Sections $(7 \mu \mathrm{m})$ were cut using a rotary microtome (Microsystems $\mathrm{GmbH}$, Germany) and mounted on poly-L-lysine coated glass slides (ThermoScientific, Menzel\&Co KG, Germany). The sections were then stained using Masson's trichrome staining. The optical microscope was conducted to observe the new bone formation and ingrowth, and the quantitative analysis of new bone, mature bone and material residual were assessed using Image Pro Plus 6.0 software.

\section{Statistical analysis}

All data were expressed as means $\pm \mathrm{SD}$. Statistical analysis was performed by ANOVA and SNK post hoc or Kruskal-Wallis nonparametric procedure followed by Mann-Whitney U test for multiple comparisons based on the normal distribution and equal variance assumption test, using SPSS v.10.1 software (SPSS Inc, USA). Values of $p<0.05$ were considered statistically significant.

\section{Results}

\section{Characterization of n-MS and n-MS/ZN/PCL scaffolds}

Fig. 1a shows the TEM image of n-MS particles, which exhibited sphere-like particles with the size of around $200 \mathrm{~nm}$. Fig. $1 \mathrm{~b}$ shows the EDS patterns of n-MS, the peaks of $\mathrm{Mg}$ and $\mathrm{Si}$ elements were found. Fig. 1c reveals the XRD patterns of n-MS and $\mathrm{ZN}$, the wide peaks at $2 \theta=20-30^{\circ}$ were found in both $\mathrm{n}-\mathrm{MS}$ and $\mathrm{ZN}$, indicating amorphous phase. Fig. $2 \mathrm{~d}$ reveals the XRD patterns of $\mathrm{C} 0, \mathrm{C} 10$ and $\mathrm{C} 20$ scaffolds. The characteristic 

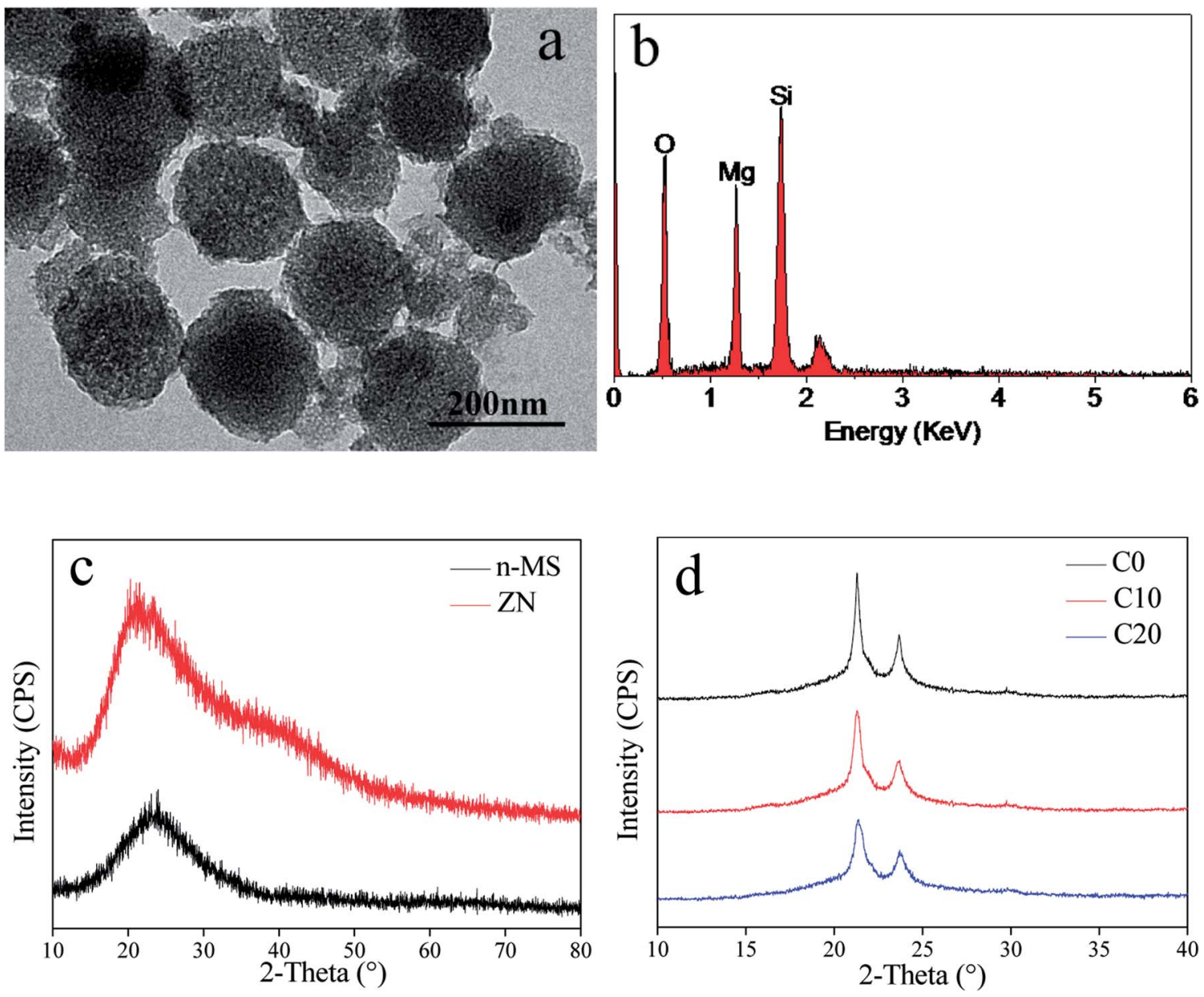

Fig. 1 TEM image (a) and EDS (b) of n-MS, and XRD (c) of n-MS and ZN, and XRD (d) of C0, C10 and C20 scaffolds.

peaks at $2 \theta=21^{\circ}$ and $23^{\circ}$ were ascribed to PCL. In addition, the characteristic peaks of PCL exhibited no obvious change with the increase of ZN content, indicating that incorporation of ZN into PCL had no significant effects on crystallinity of PCL.

The SEM micrographs of surface morphology of C0, C10 and C20 scaffolds are shown in Fig. 2. The scaffolds fabricated by 3D printing technology showed well interconnected macroporous structure and the pores sizes were around $500 \mu \mathrm{m}$. The surfaces of macropores walls of the scaffolds became rough with the increase of $\mathrm{ZN}$ content. The compressive strength and porosity of the 3D-printed scaffolds were shown in Table 2. It was found that the compressive strength of $\mathrm{C} 0$, C10 and C20 scaffolds was 7.1 MPa, 6.8 MPa and 6.5 MPa. Moreover, the porosity of $\mathrm{C} 0, \mathrm{C} 10$ and C20 scaffolds was $71.4 \%, 71.6 \%$ and $72.1 \%$, no obvious difference in porosity for these scaffolds was found.

\section{Apatite-mineralization of scaffolds}

As shown in Fig. 3 (a-f), some sphere-like apatite with the size of several micrometers were found to deposit on the surfaces of C0, C10 and C20 scaffolds after immersed into SBF for 5 days. In addition, the amounts of apatite on the C20 scaffolds were more than $\mathrm{C} 10$ and $\mathrm{C} 0$ scaffolds. The peaks of $\mathrm{Ca}$ and $\mathrm{P}$ were shown on C20 scaffold by EDS after immersed in SBF for 5 days (Fig. 3g), further confirming that the apatite was deposited on C20 scaffold surface. The ratio of $\mathrm{Ca}$ to $\mathrm{P}$ of the deposited apatite was 1.62, which was closed to hydroxyapatite of $1.67 .{ }^{23}$ The concentration of $\mathrm{Ca}$ and $\mathrm{P}$ ions gradually decreased with time (Fig. 3h) as the formation of apatite obviously consumed large amounts of $\mathrm{Ca}$ and $\mathrm{P}$ ions, while the $\mathrm{Si}$ and $\mathrm{Mg}$ ions concentrations kept increasing because of dissolution of composites scaffolds, which released Si and Mg.

\section{Degradation of scaffolds in vitro}

The weight loss (degradation) of the scaffolds in PBS increased with time (Fig. 4a). In addition, the weight loss of C20 was significantly higher than $\mathrm{C} 10$ and $\mathrm{C} 0$ scaffold. The results indicated that the degradability of scaffolds increased with ZN content in the scaffolds $(\mathrm{C} 20>\mathrm{C} 10>\mathrm{C} 0)$. The $\mathrm{pH}$ values of the PBS solution for both C0 and C10 scaffold obviously increased with time (Fig. 4b), and the increase of $\mathrm{pH}$ for $\mathrm{C} 0$ was higher than C10 scaffold. However, no obvious change of pH for C20 scaffold during immersing time. At 84 days, the $\mathrm{pH}$ values of the solution for C20, C10 and C0 scaffolds were 7.47, 7.71 and 7.83. 

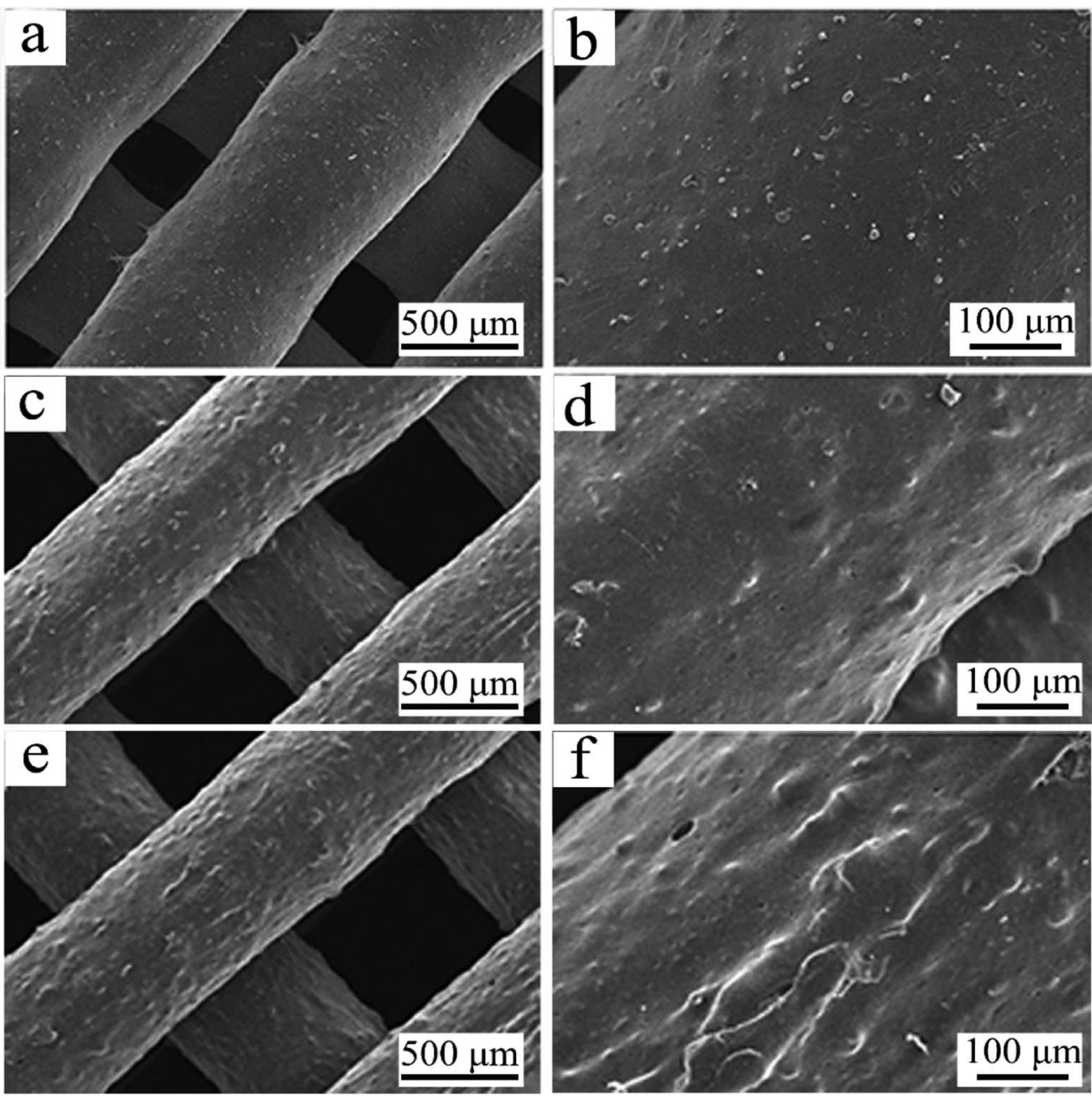

Fig. 2 SEM micrographs of CO ( $a$ and b), C10 ( $c$ and d) and C20 (e and f) scaffolds under different magnification.

Table 2 Porosity and compressive strength of 3D-printed scaffolds

\begin{tabular}{lll}
\hline Samples & Porosity $(\%)$ & Compressive strength (MPa) \\
\hline C0 & $71.4+3.2$ & $7.1+1.8$ \\
C10 & $71.6+2.8$ & $6.8+2.1$ \\
C20 & $72.1+2.3$ & $6.5+2.0$ \\
\hline
\end{tabular}

Cell morphology, proliferation and ALP activity of cells on scaffolds

The MC3T3-E1 cells were found to grow into macropores of the three kinds of scaffolds as shown in Fig. 5. Moreover, the amounts of the cells on C20 were obviously higher than those of C10 and C0 scaffolds, and C10 was more than C0 scaffold.

Fig. 6a reveals the OD values (cell proliferation) of MC3T3-E1 cells cultured on the scaffolds for 1, 3 and 5 days. The OD values of the cells on the three kind scaffolds increased with the increase of time, indicating that the cells could proliferate with time (good cytocompatibility of the scaffolds). At 1, 3 and 5 days, the OD values for $\mathrm{C} 20$ and $\mathrm{C} 10$ scaffolds were higher than $\mathrm{C} 0$, revealing that $\mathrm{C} 20$ and $\mathrm{C} 10$ scaffolds containing $\mathrm{ZN}$ promoted cells proliferation (no obvious difference between C20 and C10). In addition, at 3 and 5 days, the OD values of cells for C20 scaffold was significantly higher than C10 scaffold, and C10 was higher than C0 scaffold, showing that the OD values of cells increased with the increase of $\mathrm{ZN}$ content in scaffolds, and C20 scaffold exhibited the highest ability to promoted cells proliferation. Fig. $6 \mathrm{~b}$ shows the ALP activity of the cells cultured on the scaffolds for different time. At 7 and 10 day, the ALP activity of the cells for $\mathrm{C} 20$ was significantly higher than $\mathrm{C} 10$ and C0 scaffolds while no obvious difference for C10 and C0 scaffolds was found, indicating that $\mathrm{C} 20$ containing more $\mathrm{ZN}$ improved cells differentiation. At 14 days, the ALP activity of the cells for C20 was higher than C10 scaffold, and C10 was higher than C0 scaffold, indicating that ALP activity of cells increased with ZN content in the scaffolds, and C20 possessed the highest ability to promoted cells differentiation.

\section{Osteogenesis of scaffolds in vivo}

The 2D images from micro-CT after C0, C10 and C20 scaffolds implanted in vivo for different time as shown in Fig. 7. It was found that the newly formed bone tissues (NBs) in C0, C10 and C20 scaffolds increased with time. In addition, the amount of 

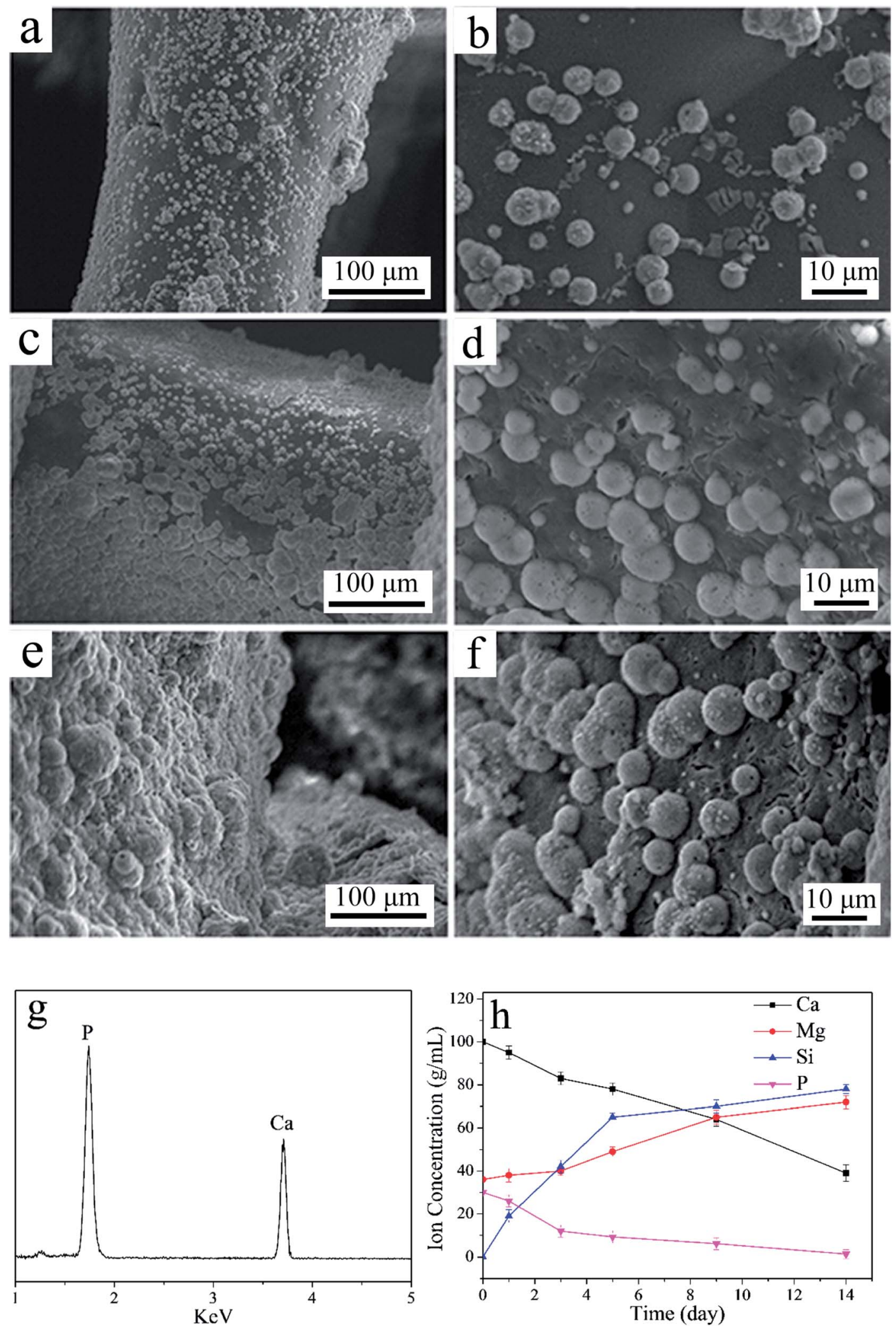

Fig. 3 SEM micrographs of C0 (a and b), C10 ( $c$ and d) and C20 (e and f) scaffolds under different magnification after immersed into SBF for 5 days, EDS (g) of C20 scaffold immersed into SBF for 5 days, and the change of ions concentrations with time (h) after C20 scaffold immersed into SBF. 

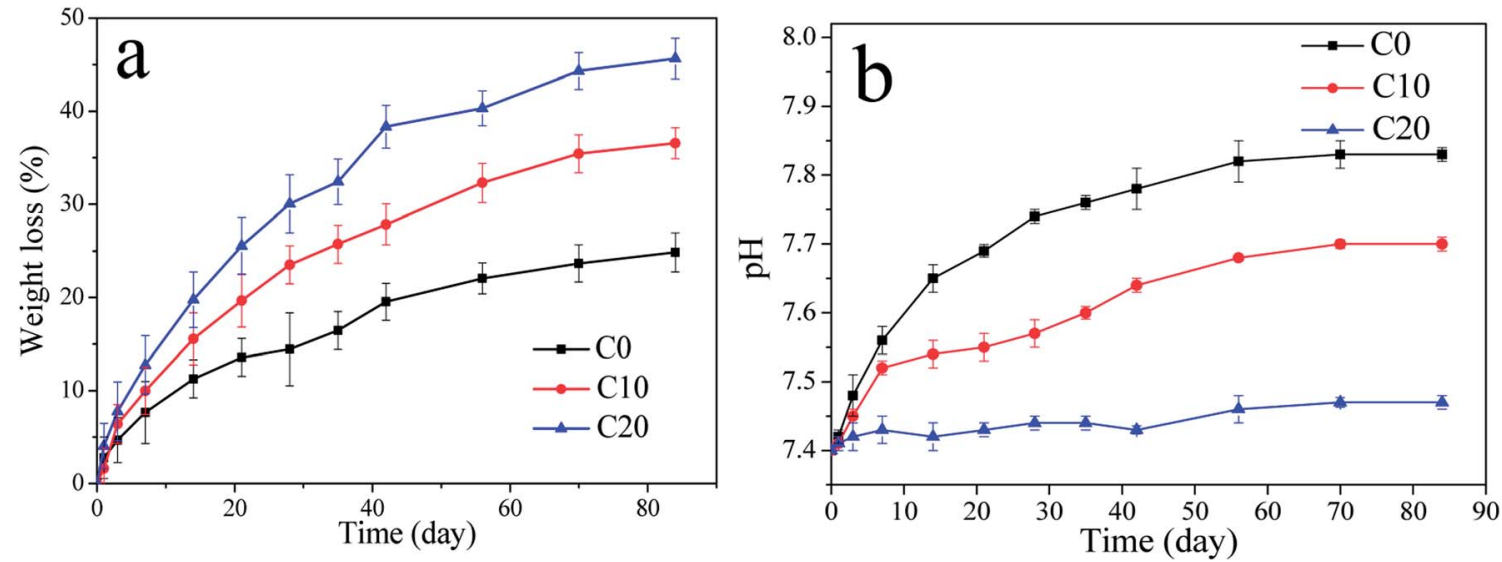

Fig. 4 Weight loss (a) of samples in PBS with time and the change of $\mathrm{pH}$ values (b) in solution with time after C0, C10 and C20 scaffolds immersed into PBS.

NBs growth into the scaffolds increased with the increase of ZN content in the scaffolds.

The histological images (Fig. 8A) revealed that the NBs were found to grow into the scaffolds, and the amounts of NBs growth into the scaffolds increased with time. Moreover, the amounts of NBs increased with the increase of ZN content in the scaffolds. The quantitative analysis of NBs area (Fig. 8B) showed that the NBs area for all scaffolds increased with time, and the NBs area increased with the increase of $\mathrm{ZN}$ content in the scaffolds. Accordingly, the material residual for all scaffolds decreased with time (Fig. 8C), and the decrease of material residual with the increase of ZN content in the scaffolds.

\section{Discussions}

In bone tissue engineering application, concisely control of the architectures of the porous scaffolds (e.g. pore size, porosity, pore morphology and pore interconnectivity, etc.) would benefit

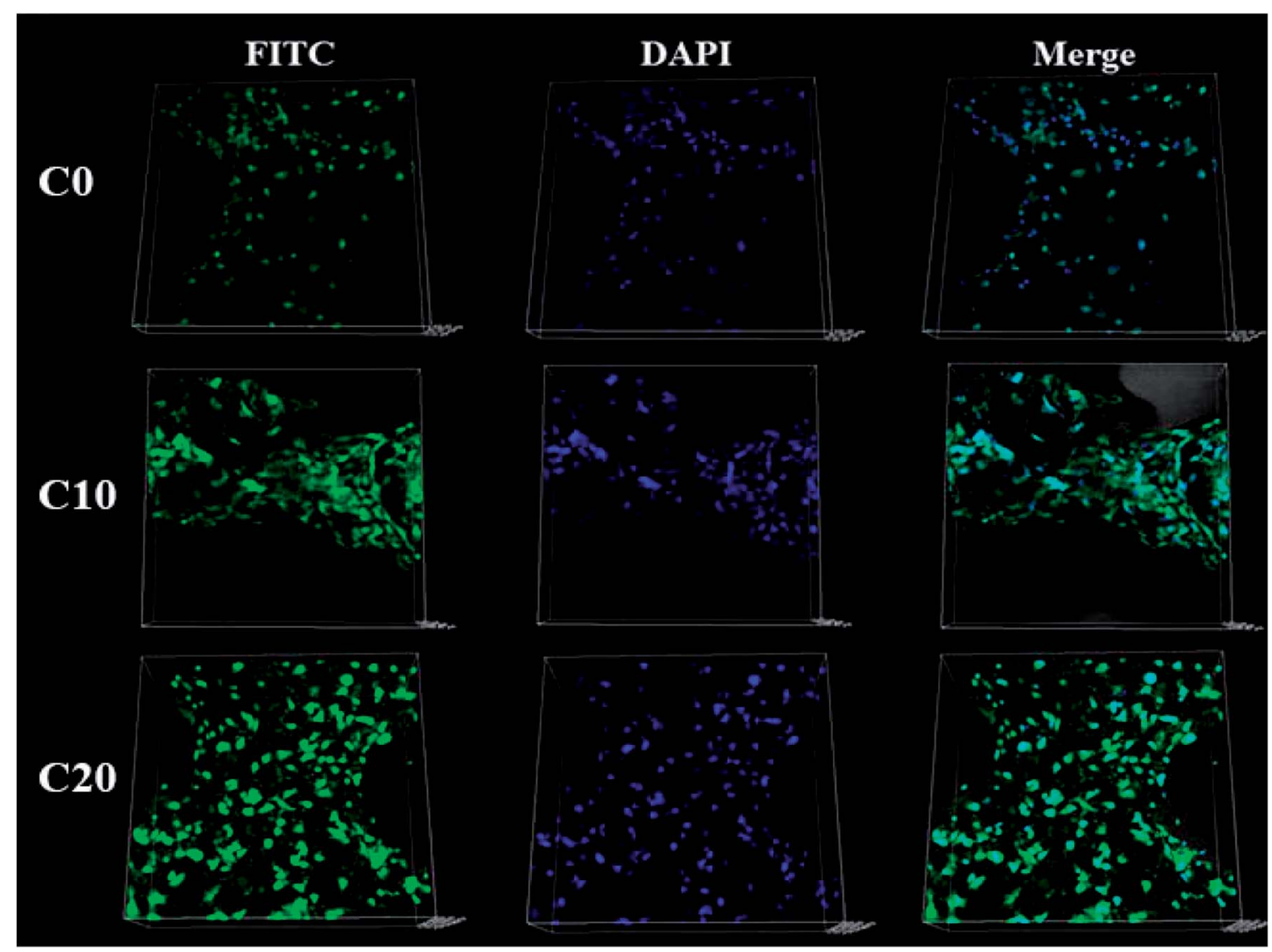

Fig. 5 CLSM images of the MC3T3-E1 cells cultured on C0, C10 and C20 scaffolds for $12 \mathrm{~h}$. 

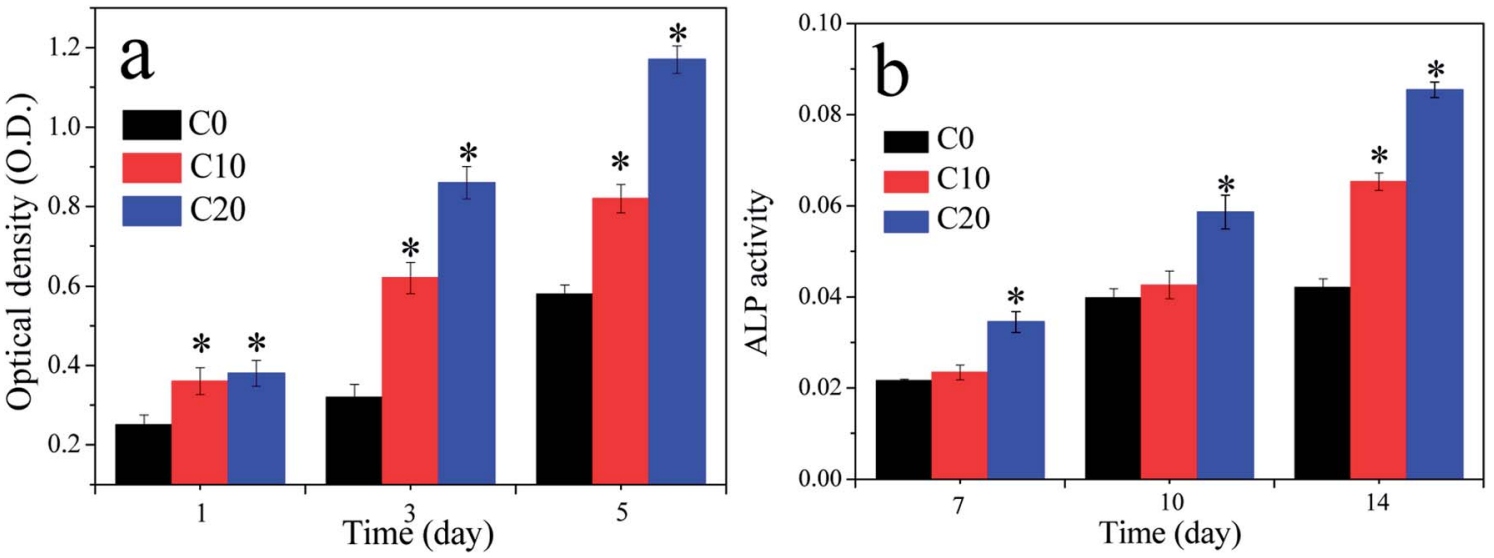

Fig. 6 Optical density (OD) values (a) and ALP activity (b) of the cells cultured on C0, C10 and C20 scaffolds for different time, * $p<0.05$.

for cells/tissues ingrowth and nutrient delivery. ${ }^{17,20}$ In this study, bioactive and degradable scaffolds of n-MS/ZN/PCL ternary composites were developed by using 3D-printing technology. The results showed that the pore morphology, pore interconnection, pore size as well as porosity of the $(\mathrm{C} 0, \mathrm{C} 10$ and C20) scaffolds could be controlled by using 3D-printing technique, and the highly interconnected scaffolds possessed the controllable macropores size of around $500 \mu \mathrm{m}$ and porosity of around $70 \%$. In addition, compared with $\mathrm{C} 0$ without $\mathrm{ZN}$ (smooth), the surfaces of macroporous walls of C10 and C20 scaffolds containing $\mathrm{ZN}$ became rough. Moreover, no obvious differences in compressive strength of the $\mathrm{C} 0, \mathrm{C} 10$ and $\mathrm{C} 20$ scaffolds were found, indicating that the addition of $\mathrm{ZN}$ did not obviously affect the compressive strength of the 3D-printed scaffolds.

Apatite mineralization on the biomaterial surface in simulated body fluid (SBF) is a generally accepted way to evaluate the in vitro bioactivity, which is also usually utilized to predict the
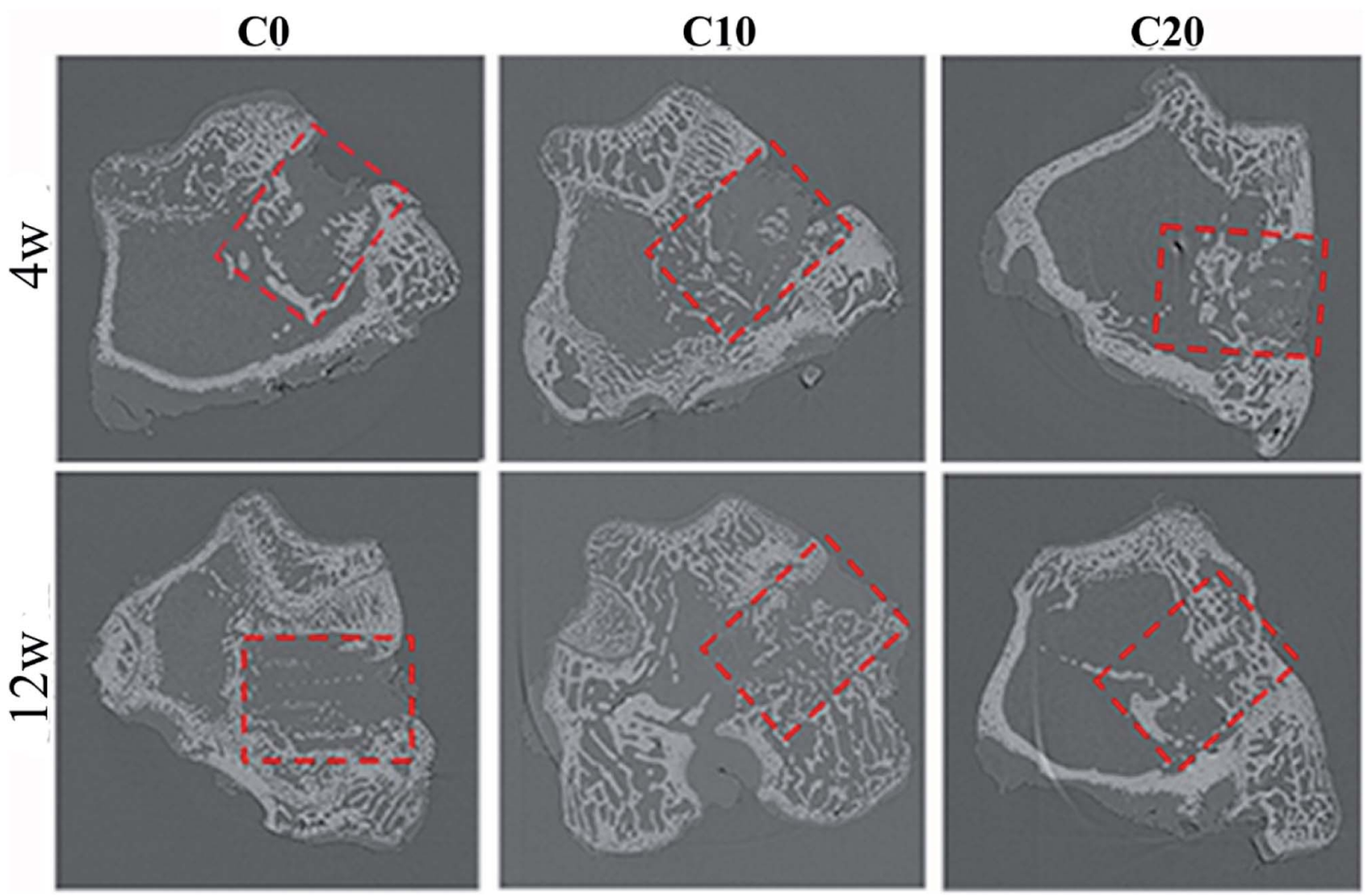

Fig. 7 The 2D images of bone-scaffolds from micro-CT after C0, C10 and C20 scaffolds implanted into femoral defects of rabbits at 4 and 12 weeks. 

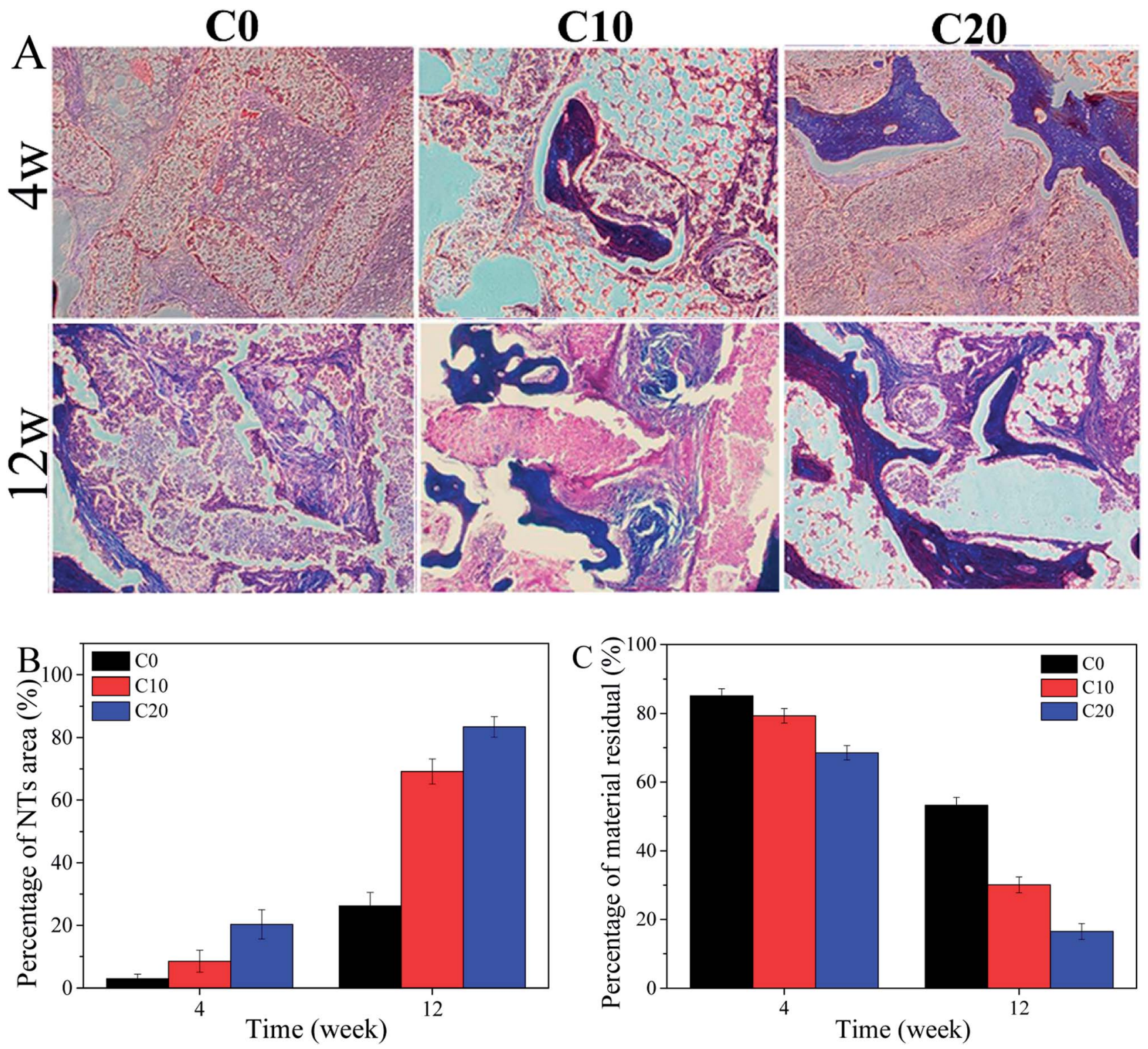

Fig. 8 Images of histological sections ( $\times 100$, Masson staining) (A), percentage of NBs area (B) and material residual (C) after C0, C10 and C20 scaffolds implanted into femoral defects of rabbits for 4 and 12 weeks.

bioactivity (osteogenic activity) of the biomaterial in vivo. ${ }^{21}$ In this study, the results suggested that the apatite mineralization ability of the scaffolds increased with the increase of $\mathrm{ZN}$ content, indicating that incorporation of ZN into the scaffolds could improve their apatite mineralization ability. The $\mathrm{C} 20$ scaffolds exhibited the highest apatite mineralization ability, indicating the best bioactivity. ${ }^{22}$ The mechanism of apatite mineralization on the scaffolds was due to the presence of $n$ MS, which was similar to the apatite mineralization on n-MS as previous publications. ${ }^{23}$ Moreover, it can be suggested that the first degradation of $\mathrm{ZN}$ (due to fast degradation rate of $\mathrm{ZN}$ ) might improve the surface area of the scaffolds with more n-MS exposing on their surfaces, which further promoted the apatite mineralization. ${ }^{24}$ Furthermore, the first degradation of ZN as well as n-MS particles exposing on the macroporous walls formed rough surfaces on the scaffolds that might offer more nucleation sites for apatite mineralization. ${ }^{25}$ The C20 containing more amounts of ZN than C10 scaffold. Therefore, the C20 scaffold exhibited the highest apatite mineralization ability.

Ideal scaffolds for bone tissue engineering should possess the matched speed of degradation with the formation of new bone when implanted in vivo. ${ }^{26}$ In this study, the results showed that incorporation of $\mathrm{ZN}$ into the scaffolds was conducive to improve their degradability (increased with the increase of ZN content), in which C20 scaffold possessed the fastest degradation rate. It can be suggested that the first degradation of ZN might form some micropores on the surfaces of the scaffold, which increased the surface area of the scaffolds. ${ }^{27}$ Therefore, incorporation of $\mathrm{ZN}$ promoted the degradation of the scaffold, which increased with the increase of $\mathrm{ZN}$ content. The $\mathrm{pH}$ values 
in PBS for both C0 and C10 obviously increased with time while no obvious change for $\mathrm{C} 20$ scaffolds. The increase of $\mathrm{pH}$ for $\mathrm{C0}$ in PBS was due to the degradation of n-MS in the scaffolds, which produced alkaline products, and formed alkaline microenvironment (micro-environment too alkaline to cells growth). ${ }^{28}$ However, the degradation of $\mathrm{ZN}$ in both $\mathrm{C} 10$ and $\mathrm{C} 20$ produce acidic products (e.g. amino acid) ${ }^{29}$ which would neutralize the alkaline products from the degradation of n-MS. The amounts of $\mathrm{ZN}$ in $\mathrm{C} 20$ were more than $\mathrm{C} 10$, which would produce more acidic products (neutralize the alkaline products from n-MS), and formed a micro-environment of $\mathrm{pH}$ value (7.47) similar to biological environment. Thus C20 scaffolds showed no change of $\mathrm{pH}$ value compared with initial value. The results indicated that incorporation of $\mathrm{ZN}$ into the scaffolds could regulate the local $\mathrm{pH}$ value in degradable solution, which could avoid too high $\mathrm{pH}$ to cells growth. ${ }^{30}$

Ideal scaffolds for bone regeneration should be able to stimulate cells proliferation, which is closely associated with new bone formation in vivo. ${ }^{31}$ The results in the present study verified that the proliferation of MC3T3-E1 cells on the scaffolds significantly enhanced with the increase of ZN content, and C20 scaffold exhibited the highest ability to promoted cells proliferation. In addition, the results from CLSM images indicated that the cells grew into the scaffolds, and the amounts of the cells in C20 scaffold were more than C10 and C0 scaffolds, indicating that the $\mathrm{C} 20$ scaffolds not only stimulated cells proliferation but also promoted cells growth into the scaffolds. ${ }^{19}$ ALP activity is generally regarded as an early marker of the differentiation of osteoblasts, and an indicator of osteogenesis in vivo. ${ }^{32}$ The present study showed that the ALP activity of cells on C20 was obviously higher than C10 scaffolds, and C10 was higher than C0 scaffolds. It could be suggested that the amounts of $\mathrm{ZN}$ in the scaffolds had obvious effects on ALP activity of cells. Overall, the C20 scaffolds significantly promoted cell proliferation and osteogenic differentiation, indicating good cytocompatibility. ${ }^{19}$

Previous studies have shown that the release of ions (e.g. Si, $\mathrm{Mg}$, etc.) from bioactive scaffolds was a key factor that influenced the cell responses (e.g. cells proliferation and differentiation, etc..$^{33}$ In this study, the $\mathrm{Si}$ and $\mathrm{Mg}$ ions were found to release from $\mathrm{C} 20$ scaffold into SBF due to the dissolution of nMS in the scaffold. Therefore, the improvements of cell proliferation and ALP activity of MC3T3-E1 cells were attributed to the release of these ions from the scaffolds. Moreover, the first degradation of $\mathrm{ZN}$ in $\mathrm{C} 20$ would lead to further degradation of n-MS (surface area of C20 increase), which released more Si and $\mathrm{Mg}$ ions (C20 > C10) in the micro-environment to stimulate cells responses. ${ }^{24}$ In addition, the formation of rough surface of the C20 scaffold due to the increase of $\mathrm{ZN}$ content (compared with $\mathrm{C} 10$ and $\mathrm{C} 0)$ might be useful for the cells responses (e.g. cell adhesion). ${ }^{34}$ Furthermore, the formation of apatite on the scaffolds might stimulate the cells responses (e.g. cell differentiation). ${ }^{35}$

As a scaffold for bone regeneration, it is critical to investigate its osteogenic ability in vivo using animal models. ${ }^{36}$ In the present study, the osteogenic ability of the scaffolds implanted in vivo was studied by micro-CT and histological analysis. The results confirmed that the newly formed bone tissues (NBs) in the scaffolds increased with the increase of $\mathrm{ZN}$ content, and $\mathrm{C} 20$ possessed the best osteogenic ability, indicating that ZN played key roles on improvement the osteogenic ability of the scaffolds. In addition, the histological images and quantitative analysis demonstrated that the NBs grew into the scaffolds, and the amounts of NBs increased with the increase of ZN content, in which the C20 possessed the highest new bone formation ability. Accordingly, the material residual for the scaffolds decreased with the increase of ZN content, and C20 exhibited the lowest material residual, indicating good degradability. The mechanism of degradation of scaffolds in vivo was different from in vitro. The mechanism of degradation of scaffolds in vitro was the dissolution. However, the mechanism of degradation of scaffolds in vivo included two factor: dissolution and cellmediated degradation (biodegradation). ${ }^{37}$ It could be suggested that incorporation of ZN into scaffolds not only significantly improved the osteogenic ability but also promoted the degradability of the scaffolds in vivo. ${ }^{38}$

The generally accepted mechanism of new bone formation in the bioactive scaffold assumed that osteoblasts first adhered to the scaffold surface, formed bone matrix, then mineralized to form bone tissues. ${ }^{39}$ The process of bone formation was favored by the apatite-mineralization on the scaffold in a physiological environment because the mineralized apatite (closely resembles bone mineral in composition and structure) might serve as a foundation for osteogenesis. ${ }^{\mathbf{4 0}}$ In this study, apatitemineralization ability of the scaffolds increased with the ZN content, and C20 scaffold exhibited the highest apatitemineralization ability. Therefore, the improvement of new bone formation ability for C20 scaffold was ascribed to the enhancement of apatite-mineralization ability of the scaffold, which increased with $\mathrm{ZN}$ content. In addition, previous studies have revealed that the $\mathrm{Mg}$ and $\mathrm{Si}$ ions release from bioglass/ ceramics could not only stimulate the proliferation and differentiation of osteoblasts and hBMSCs in vitro but also promoted new bone formation in vivo. ${ }^{41}$ Therefore, the enhancement of osteogenesis of C20 in vivo might be attributed to the release of $\mathrm{Mg}$ and Si ions from the scaffolds. In summary, the in vitro and in vivo results indicated that the excellent biocompatibility, degradability and osteogenesis of the scaffolds of n-MS/ZN/PCL ternary composites were attributed to the bioperformances of both the n-MS and ZN together in the scaffolds, and the addition of $\mathrm{ZN}$ into the scaffolds might be an effective strategy to improve the bioperformance of 3D-printed scaffolds.

\section{Conclusions}

Bioactive and degradable scaffolds of n-MS/ZN/PCL ternary composites were developed by 3D-printing method for bone tissue engineering applications. The results showed that the scaffolds possessed well interconnected macropores of around $500 \mu \mathrm{m}$ and controllable porous structures. The in vitro apatite mineralization ability and degradability of the scaffolds were greatly improved with the increase of ZN content, in which C20 scaffold possessed the highest apatite mineralization ability and the fastest degradability. The scaffolds containing ZN 
significantly promoted the proliferation and early differentiation of MC3T3-E1 cells, which depended on the content of ZN. Furthermore, the in vivo osteogenesis of the 3D-printed scaffolds was obviously enhanced, which depended on the content of $\mathrm{ZN}$. The results demonstrated that the ternary composites scaffolds combined with advantages of both n-MS and ZN could effectively promote bone regeneration. The C20 scaffold prepared by the $3 \mathrm{D}$-printing method had a great potential for bone tissue engineering applications.

\section{Conflicts of interest}

There are no conflicts to declare.

\section{Acknowledgements}

The grants were from the National Natural Science Foundation of China (81772343), and the Research Project of Jiangsu Provincial Health and Family Planning Commission (H201662).

\section{Notes and references}

1 J. Zhang, S. Zhao, Y. Zhu, Y. Huang, M. Zhu, C. Tao and C. Zhang, Acta Biomater., 2014, 10, 2269-2281.

2 C. M. Curtin, G. M. Cunniffe, F. G. Lyons, K. Bessho, G. R. Dickson, G. P. Duffy and F. J. O'Brien, Adv. Mater., 2012, 24, 749-754.

3 S. Sprio, A. Tampieri, G. Celotti and E. Landi, J. Mech. Behav. Biomed. Mater., 2009, 2, 147-155.

4 Q. Fu, M. N. Rahaman, B. S. Bal, R. F. Brown and D. E. Day, Acta Biomater., 2008, 4, 1854-1864.

5 J. A. Sowjanya, J. Singh, T. Mohita, S. Sarvanan, A. Moorthi, N. Srinivasan and N. Selvamuruqan, Colloids Surf., B, 2013, 4, 294-300.

6 T. Qu and X. Liu, J. Mater. Chem. B, 2013, 1, 4764-4772.

7 C. Isikli, V. Hasirci and N. Hasirci, J. Tissue Eng. Regener. Med., 2012, 6, 135-143.

8 H. T. Oyama, D. Tanishima and S. Maekawa, Polym. Degrad. Stab., 2016, 134, 265-271.

9 E. J. Chong, T. T. Phan, I. J. Lim, Y. Z. Zhang, B. H. Bay, S. Ramakrishna and C. T. Lim, Acta Biomater., 2007, 3, 321-330.

10 F. Wu, J. Wei, C. S. Liu, B. O'Neil and Y. Ngothai, Composites, Part B, 2012, 43, 2192-2197.

11 Y. Miao, R. Yang, D. Y. B. Deng and L. M. Zhang, RSC Adv., 2017, 7, 17711-17719.

12 E. Corradini, P. S. Curti, A. B. Meniqueti, A. F. Martins, A. F. Rubira and E. C. Muniz, Int. J. Mol. Sci., 2014, 15, 22438-22470.

13 M. Demir, L. Ramos-Rivera, R. Silva, S. N. Nazhat and A. R. Boccaccini, J. Biomed. Mater. Res., Part A, 2017, 105, 1656-1665.

14 S. Babitha and P. S. Korrapati, Biomed. Mater., 2017, 12, 055008.

15 Y. Zhang, N. Cheng, R. Miron, B. Shi and X. Cheng, Biomaterials, 2012, 33, 6698-6708.
16 B. Rais, M. Koster, M. I. Rahim, M. Pils, J. M. Seitz, H. Hauser, D. Wirth and P. P. Mueller, J. Biomed. Mater. Res., Part A, 2016, 104, 2149-2158.

17 C. H. Zhang, D. Cheng, T. H. Tang, X. L. Jia, Q. Cai and X. P. Yang, J. Mater. Chem. B, 2015, 3, 5300-5309.

18 C. Kascholke, S. Hendrikx, T. Flath, D. Kuzmenka, H. M. Dorfler, D. Schumann, M. Gressenbuch, F. P. Schulze, M. Schulz-Siegmund and M. C. Hacker, Acta Biomater., 2017, 63, 336-349.

19 Y. L. Li, Y. Xiao and C. S. Liu, Chem. Rev., 2017, 117, 43764421.

20 J. A. Inzana, D. Olvera, S. M. Fuller, J. P. Kelly, O. A. Graeve, E. M. Schwarz, S. L. Kates and H. A. Awad, Biomaterials, 2014, 35, 4026-4034.

21 A. M. Deliormanli and M. N. Rahaman, J. Eur. Ceram. Soc., 2012, 32, 3637-3646.

22 A. Rifai, N. Tran, D. W. Lau, A. Elboutne, H. L. Zhan, A. D. Stacey, E. L. H. Mayes, A. Sarker, E. P. Ivanova, R. J. Crawford, P. A. Tram, B. C. Gibson, A. D. Greentree, E. Pirogova and K. Fox, ACS Appl. Mater. Interfaces, 2018, 10, 8474-8484.

23 T. W. Sun, W. L. Yu, Y. J. Zhu, R. L. Yang, Y. Q. Shen, D. Y. Chen, Y. H. He and F. Chen, ACS Appl. Mater. Interfaces, 2017, 9, 16435-16447.

24 Z. R. Gu, S. C. Wang, W. Z. Weng, X. Chen, L. H. Cao, J. Wei, J. W. Shin and J. C. Su, Mater. Sci. Eng., C, 2017, 75, 620-628. 25 Z. Y. Wu, Q. Li, Y. K. Pan, Y. Yao, S. C. Tang, J. C. Su, J. W. Shin, J. Wei and J. Zhao, Int. J. Nanomed., 2017, 12, 3637-3651.

26 L. Krishnan, L. B. Priddy and C. Esancy, Acta Biomater., 2016, 49, 101-112.

27 Z. Fereshteh, M. Fathi, A. Bagri and A. R. Boccaccini, Mater. Sci. Eng., C, 2016, 68, 613-622.

28 Z. Y. Wu, K. Zheng, J. Zhang, T. T. Tang, H. Guo, A. R. Boccaccini and J. Wei, J. Mater. Chem. B, 2016, 4, 7974-7988.

29 X. Chen, D. S. Brauer, N. Karpukhina, R. D. Waite, M. Barry, I. J. Mckay and R. G. Hill, Mater. Lett., 2014, 126, 278-280.

30 H. J. Wang, S. J. Gong, Z. X. Lin, J. X. Fu, S. T. Xue, J. C. Huang and J. Y. Wang, Biomaterials, 2007, 28, 3952-3964.

31 E. Ko, K. Yang, J. Shin and S. W. Cho, Biomacromolecules, 2013, 14, 3202-3213.

32 H. M. Zhang, J. H. Wang, F. Deng, E. Y. Huang, Z. J. Yan, Z. L. Wang, Y. L. Deng, Q. Zhang, Z. L. Zhang, J. X. Ye, M. Qiao, R. F. Li, J. Wang, Q. Wei, G. L. Zhou, H. H. Lu, R. C. Haydon, T. C. He and F. Deng, Biomaterials, 2015, 39, 145-154.

33 C. T. Wu, R. Miron, A. Sculean, S. Kaskel, T. Doert, R. Schulze and Y. F. Zhang, Biomaterials, 2011, 32, 7068-7078.

34 L. Cai, Y. K. Pan, S. C. Tang, Q. Li, T. T. Tang, K. Zheng, A. R. Boccaccini, S. C. Wei, J. Wei and J. C. Su, J. Mater. Chem. B, 2017, 5, 8337-8352.

35 G. Pezzotti, N. Oba, W. L. Zhu, E. Marin, A. Rondinella, F. Boschetto, B. McEntire, K. Yamamoto and B. S. Bal, Acta Biomater., 2017, 64, 411-420. 
36 Q. Q. Yao, J. G. L. Cosme, T. Xu, J. M. Miszuk, P. H. S. Picciani, H. Fong and H. L. Sun, Biomaterials, 2017, 115, 115-127.

37 A. J. Drelich, S. Zhao, R. J. Guillory, J. W. Drelich and J. Goldman, Acta Biomater., 2017, 58, 539-549.

38 P. Y. Zhou, Y. Xia, X. S. Cheng, P. F. Wang, Y. Xie and S. G. Xu, Biomaterials, 2014, 35, 10033-10045.
39 S. Cao, H. J. Li, K. Z. Li, J. H. Lu and L. L. Zhang, J. Biomed. Mater. Res., Part A, 2016, 104, 533-543.

40 M. C. Xu, D. Zhai, J. Chang and C. T. Wu, Acta Biomater., 2014, 10, 463-476.

41 M. C. Tousi, M. F. Velten, T. J. Bishop, K. K. Leong, N. S. Barkhordar, G. W. Marshall, P. M. Loomer, P. B. Aswath and V. G. Varanasi, Mater. Sci. Eng., C, 2013, 33, 2757-2765. 